\title{
ABORDAGEM CLÍNICO-CIRÚRGICA DE DESVIO PORTOSSISTÊMICO CONGÊNITO EM PEQUENOS ANIMAIS: quais as novidades?
}

\section{Clinical and surgical approach of congenital portosystemic shunt in small animals: are there new informations?}

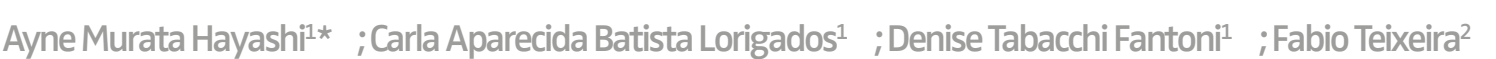 \\ Márcio Antonio Brunetto ${ }^{\mathbf{3}}$; Ana Carolina Brandão de Campos Fonseca Pinto ${ }^{\mathbf{D}}$; Julia Maria Matera ${ }^{1}$ \\ *Autor Correspondente: Ayne Murata Hayashi. Av. Prof. Orlando Marques de Paiva, 87, \\ São Paulo, SP, Brasil, CEP: 05508-270. E-mail: aynevet@usp.br
}

\begin{abstract}
T Como citar: HAYASHI, Ayne Murata et al. Abordagem clínico-cirúrgica de desvio portossistêmico congênito em pequenos animais: quais as novidades? Revista de Educação Continuada em Medicina Veterinária e Zootecnia do CRMV-SP, São Paulo, v.18, n. 2, 2020. Doi 10.36440/recmvz.v18i2.38043

Cite as: HAYASHI, Ayne Murata et al. Clinical and surgical approach of congenital portosystemic shunt in small animals: are there new informations? Journal of Continuing Education in Animal Science of CRMV-SP, São Paulo, v.18, n.2, 2020. Doi 10.36440/recmvz.v18i2.38043
\end{abstract}

\section{Resumo}

Foi realizada uma revisão sobre a fisiopatogenia, sinais clínicos, diagnóstico e principais tratamentos e técnicas para o desvio portossistêmico em pequenos animais. As fontes pesquisadas foram: CAB, MEDILINE por um período retrospectivo de 20 anos e acervos da Biblioteca da Faculdade de Medicina Veterinária e Zootecnia (FMVZ) da Universidade de São Paulo (USP). O desvio portossistêmico congênito (DPSC) é uma das anormalidades vasculares mais comuns em cães, as raças de pequeno porte apresentam maior incidência. Os DPSCs em cães e gatos são comunicações vasculares que ocorrem do sistema venoso portal para o sistema venoso sistêmico, ou seja, fazem uma via secundária. Há dois tipos de DPSCs, intra-hepático e extra-hepático, observados com frequência em raças de grande porte e miniaturas, respectivamente. 0 diagnóstico é baseado no histórico de animais jovens com retardo de crescimento, letargia, convulsão ou distúrbio de comportamento principalmente após alimentação, retorno demorado de anestesia ou sedação, crise de encefalopatia hepática, em raças predispostas. A confirmação do vaso anômalo é realizada pela ultrassonografia com doppler, angiografia por tomografia computadorizada ou por ressonância magnética. 0 manejo clínico pode aliviar sinais clínicos de encefalopatia hepática, porém em longo prazo, é questionável, pois o fluxo sanguíneo portal

1 Departamento de Cirurgia, Faculdade de Medicina Veterinária e Zootecnia da Universidade de São Paulo (FMVZ-USP), São Paulo, SP, Brasil

2 Departamento de Clínica Médica, Faculdade de Medicina Veterinária e Zootecnia da Universidade de São Paulo (FMVZ-USP), São Paulo, SP, Brasil

3 Departamento de Nutrição e Produção Animal, Faculdade de Medicina Veterinária e Zootecnia da Universidade de São Paulo (FMVZ-USP), São Paulo, SP, Brasil 
continua desviado para a circulação sistêmica, comprometendo a distribuição de fatores hepatotróficos ao fígado e ocorrendo uma contínua atrofia do mesmo. 0 único tratamento definitivo é o cirúrgico, idealmente com técnica que atenue progressivamente o vaso anômalo e evite a hipertensão portal aguda. Dentre as técnicas de tratamento cirúrgico descritas o uso de ameroide constrictor apresenta-se como uma das formas mais seguras a obtenção do fechamento do desvio. Entretanto, os portadores de DPSC podem sofrer de complicações perioperatórias graves e necessitam de abordagem clínicocirúrgica específica, associado a um diagnóstico por imagem precisa para a correta localização do vaso anômalo e sucesso cirúrgico.

Palavras-chave: Anomalia vascular hepática. Ameroide constrictor. Encefalopatia hepática. Hipertensão portal. Tomografia computadorizada.

\section{Abstract}

It was performed a review of pathophysiology, clinical signs, diagnostic and main treatments and techniques for portosystemic shunts in small animals. The researched Sources were: a 20 years retrospective research of CAB, MEDILINE and collection of Faculty of MedicineVeterinary and Animal Science (FMVZ) of the Universidade de São Paulo (USP). Congenital portossystemic shunts (CPSS) are one of the vascular abnormalities more common in dogs. Small breeds are the most affected. The CPSS in dogs and cats, are vascular communications between portal venous system and systemic venous system, in other words, a secondary via. There are two types of CPSS, the intrahepatic and extrahepatic shunts, observed in large and small breeds, respectively. The diagnosis is based on clinical findings of young dogs referring delayed development, lethargy, convulsions, behavior disturbances mainly after food ingestion, poor recovery after anesthesia or sedation, hepatic encephalopathy crisis observed breeds with predisposition. Definitive diagnosis of CPSS is done by abdominal doppler ultrasonography, computed tomography angiography or magnetic resonance. Medical management can alleviate signs of hepatic encephalopathy. However, long term treatment is questionable because blood circulation is still bypassing liver to systemic circulation, leading to disturbance of hepatotropic factors distribution to the liver, resulting in liver atrophy. The only definitive treatment is the surgical one. The ideal technique is the progressive attenuation of anomalous vessel and avoid acute portal hypertension. Within the described techniques for surgical treatment, the use of ameroid constrictor represents the safest way of vessel closure. On the other way, some CPS patients can suffer severe perioperative complications. So they need specific clinical and surgical approaches associated to precise image diagnostic to correct localization of the anomalous vessel and surgical success.

Keywords: Hepatic vascular anomalies. Portal hypertension. Ameroid constrictor. Hepatic encephalopathy. Computed tomography.

\section{Introdução}

Atualmente tem sido observado um crescente aumento do número de pacientes com anomalias vasculares hepáticas na clínica de pequenos animais, provavelmente devido a um maior conhecimento e melhora no diagnóstico por imagem e como consequência o estabelecimento de tratamentos adequados. 0 presente trabalho analisa o manejo clínico e o tratamento cirúrgico de tal patologia com técnicas que permitem a atenuação do vaso anômalo para evitar uma hipertensão portal aguda.

Os desvios portossistêmicos congênitos (DPSCs) são anomalias vasculares onde há conexão da circulação portal com a sistêmica, afetando o metabolismo hepático e acarretando diversos sinais clínicos importantes, frequentemente necessitando de atendimento emergencial (TOBIAS, 2007). Os portadores de DPSCs manifestam sinais relacionados a disfunção hepática, no entanto, alguns são assintomáticos e ao apresentarem microhepatia ou hipoalbuminemia é estabelecida a suspeita da afecção (WOLSCHRIJN et al., 2000; MEHL et al., 2005; RADINSKY, 2013). 
Nos casos de DPSCs os quadros hemorrágicos espontâneos são incomuns. Entretanto, as hemorragias podem ser disparadas na presença concomitante de úlceras gastrointestinais, uso de fármacos anticoagulantes e nos procedimentos invasivos como as intervenções cirúrgicas, de aspiração ou biópsia (BERENT; TOBIAS, 2012). Além disso, pacientes com DPC extra-hepático podem manifestar urolitíase, predominantemente de urato de amônio (RADINSKY, 2013; CAPORALI, 2015; MANKIN, 2015).

Portanto, o tratamento cirúrgico indicado para pacientes com DPCs, pode determinar evoluções clínicas nem sempre isentas de complicações. O objetivo do presente trabalho é a avaliação das manifestações clínicas dos diferentes tipos de DPSCs submetidos a intervenção cirúrgica bem como das intercorrências ocorridas no transoperatório e pós-operatório imediato, visando a obtenção de informações de relevância clínica que auxiliem a melhor abordagem clínica-cirúrgica dos respectivos casos.

\section{Embriologia e falhas envolvidas no DPSC}

As veias da cavidade abdominal são derivadas das veias umbilical, vitelina e cardinal caudal do embrião. As veias vitelinas formam a veia hepática esquerda, sinusóides hepáticos, parte hepática da veia cava caudal e veia porta pré-hepática e seus tributários. Porções dos sistemas vitelino e umbilical se unem para dar origem ao duto venoso e ramo esquerdo da veia porta (BERENT; TOBIAS, 2012; TOBIAS, 2007). Quando ocorrem falhas no desenvolvimento resultando em comunicação funcional anormal entre estes dois sistemas cardinal e vitelino ocorrem os desvios portoázigos e portocavais extra-hepáticos congênitos e em alguns animais a veia cava caudal pode estar interrompida (BERENT; TOBIAS, 2012). No feto há numerosas comunicações portocavais e portoázigas não funcionais. Se ocorrer uma hipertensão portal crônica, essas comunicações poderão se tornar funcionais. Os desvios intra-hepáticos esquerdos se apresentam como vasos únicos e se originam pela persistência, após o nascimento, do duto venoso fetal, que normalmente no cão tem oclusão funcional dentro de dois a seis dias após o nascimento, devido a mudança na pressão sanguínea que ocorre com 0 desaparecimento do fluxo venoso umbilical (TOBIAS, 2007).

Em cães neonatos não há esfíncter e o duto vai se estreitando depois do nascimento. A proliferação de tecido conjuntivo na junção do duto venoso e seio portal umbilical expande-se até a terminação do duto na veia hepática esquerda, com oclusão estrutural em três semanas pós nascimento (TOBIAS, 2007).

Defeitos no processo de oclusão da junção duto venoso-seio portal umbilical levam a persistência do duto venoso e determinam o estabelecimento do desvio portossistêmico intrahepático esquerdo. Entretanto tal anomalia também pode surgir quando existe uma anastomose portal ou da veia hepática ou veia cava caudal. Já nos desvios centrais e do lado direito ainda não foi determinado o mecanismo do estabelecimento da anomalia, acreditando-se que possa ser decorrente da persistência de uma parte do sistema vitelino (TOBIAS, 2007; RADINSKY, 2013). 


\section{Anatomia do sistema vascular hepático de relevância cirúrgica}

O fígado apresenta dois suportes sanguíneos aferentes - um sistema portal de baixa pressão e um sistema arterial de alta pressão (RADLINSKY, 2013). 0 sistema porta abastece $75 \%$ a $80 \%$ do volume de sangue aferente e $50 \%$ do oxigênio para o fígado. 0 restante é abastecido pela artéria hepática. Os vasos tributários da veia porta, do aspecto caudal para cranial são os vasos mesentéricos que drenam o intestino delgado e formam a veia mesentérica cranial que é a maior tributária, a veia mesentérica caudal que drena o cólon e reto proximal, veia lienal que recebe sangue do baço e do estômago por meio da veia gástrica esquerda, e em cães a veia gastroduodenal que drena porções do pâncreas, duodeno e estômago (BERENT; TOBIAS, 2012).

Nos cães a veia porta divide-se em ramos portais direito e esquerdo a cerca de 0.5 a $1 \mathrm{~cm}$ da entrada da sua maior tributária - gastroduodenal. A veia porta direita é um tronco pequeno que supre o processo caudado do lobo caudado e o lobo hepático medial direito, que na região da bifurcação pode estar parcialmente ou completamente rodeado pelo parênquima hepático. A veia porta esquerda é maior e mais longa e dá origem ao ramo central para o lobo medial direito e ramo papilar pequeno para o processo papilar do lobo caudado antes de se dividir em ramo quadrado medial esquerdo e lateral esquerdo. Já em gatos há a divisão de ramos portais direito, central e esquerdo. A artéria hepática canina se divide por sua vez em ramos lateral direita, lateral esquerda e esquerdos. É encontrada geralmente no aspecto dorsal ou caudal do ramo portal esquerdo. Os ductos biliares e ramos da artéria hepática estão localizados normalmente na superfície ventral da veia porta, entretanto alguns ramos arteriais podem estar em uma posição dorsal. 0 sangue dos ramos da veia porta e artéria hepática se misturam dentro dos sinusoides hepáticos antes de entrarem nas veias centrais. As veias centrais se fundem e eventualmente formam as veias hepáticas que drenam o sangue para a porção abdominal da veia cava caudal, nos cães há cerca de cinco a oito veias formando uma espiral ao longo da veia cava caudal. A veia hepática esquerda corresponde a maior e mais cranial das veias hepáticas, terminando no aspecto lateral esquerdo da veia cava caudal, próximo a superfície visceral do diafragma. Entretanto, cerca de $1 / 3$ a $1 / 5$ da circunferência da veia hepática lateral esquerda é circundado pelo parênquima hepático ou escondido pelo ligamento triangular esquerdo (BERENT; TOBIAS, 2012).

\section{Desvio portossistêmico congênito}

Nos cães o desvio portossistêmico congênito (DPSC) é uma anormalidade vascular muito frequente cuja ocorrência é muito superior à observada nos seres humanos (BUSSADORI et al., 2008). Por outro lado, as conexões venosas anormais são descritas na maioria dos casos em cães do que em gatos. Estas anormalidades vasculares são divididas em duas categorias de acordo com sua origem, sendo DPSC derivando de falhas embriogenéticas no desenvolvimento dos sistemas venosos vitelínico e cardinal, e desvios porto-sistêmicos adquiridos (DPSA) derivando da recanalização de conexões vasculares embriogênicas vestigiais pré-existentes entre sistemas cava e portal como uma consequência de hipertensão portal (RADLINSKY, 2013; RICCIARDI, 2017).

As malformações arteriovenosas hepáticas são presumidamente outra forma de anomalias congênitas em animais. Já as fístulas arteriovenosas são adquiridas (SPECHI et al., 2018). Em seres humanos podem ocorrer fístulas secundariamente a traumas hepáticos (TOBIAS, 2007). Alguns casos de malformação arteriovenosa hepática podem ser embolizadas com cola de cianoacrilato por meio do acesso da artéria femoral guiado com fluoroscopia, um guia retrógrado avança até a aorta, sendo selecionada a artéria hepática por meio da artéria celíaca. Um cateter é direcionado sobre o guia e o angiograma é realizado. Quando a comunicação é constatada os vasos que alimentam a malformação são selecionados e a cola pode ser infundida (BERENT; TOBIAS, 2009). Também deve ser diferenciada a 
displasia hepática microvascular ou hipoplasia portal venosa, na qual os vasos portais intra-hepáticos são pequenos ou ausentes e há uma hiperplasia de arteríolas portais. Neste caso, ocorrem desvios microscópicos do sangue através do fígado e não há o desvio portossistêmico macroscópico. Atualmente o diagnóstico da displasia hepática microvascular é efetuado com o emprego do exame histopatológico (com similaridades ao shunt portossistêmico congênito) associado à ausência de desvios macroscópicos (RADINSKY, 2013).

\section{Tipos de shunts ou DPSCs}

Há dois tipos de DPSCs, intra-hepático e extra-hepático. Os desvios intra-hepáticos (DIH) são divididos de acordo com a sua localização anatômica. DIH à esquerda, apresenta geralmente um formato tubular drenando o sangue para a veia hepática esquerda. Já o DIH central é um forame entre as porções dilatadas da veia porta intra-hepática e a veia cava caudal. O DIH direito apresenta-se com um vaso grande e tortuoso (BUSSODORI et al., 2008).

Os DPSCs extra-hepáticos observados em cães de raças pequenas localizam-se em uma posição caudal ao fígado (HUNT; HUGUES, 2006). As raças mais acometidas são o bichon havanês, yorkshire terrier, maltês, dandie dinmont terrier e pugs (TOBIAS; ROHRBACH, 2003). Outras raças descritas são schnauzer miniatura, poodle, Ihasa apso, bichon frise, jack russel terrier, shih tzu, pequinês. Nos cães da raça yorkshire o DPSCs é hereditário e em outras raças também pode ser de etiologia genética (RADINSKY, 2013).

Já os DPSC intra-hepáticos são diagnosticados em cães de raças grandes como pastor alemão, golden retriever, doberman pinscher, labrador, setter irlandês, irish wolfhound, contudo também podem ser observados em poodles toy e miniatura. Nos DPSC intra-hepáticos, geralmente há um grande volume de sangue portal desviado para o vaso anômalo, o que determina o estabelecimento de sinais clínicos mais severos em idade precoce quando comparados aos DPSCS extra-hepáticos (BERENT; TOBIAS, 2012).

Em irish wolfhound, o DPSC intra-hepático esquerdo é a forma mais comum enquanto em golden, labrador e cão australiano geralmente tem DPSC intra-hepático central (SANTILLI; GERBONI, 2003).

\section{Fisiopatologia do DPC}

Muitas substâncias normalmente metabolizadas ou excretadas pelo fígado são desviadas da circulação venosa portal para o sistema venoso sistêmico (VOGT et al., 1996). Deste modo, as substâncias hepatotróficas do pâncreas, como a insulina e as substâncias hepatotróficas dos intestinos não conseguem alcançar o fígado, levando a atrofia hepática ou a falha na manutenção do tamanho normal do órgão. A consequência do processo é a insuficiência ou encefalopatia hepática, onde pode ser identificada uma síndrome neurológica, pois várias substâncias como amônia, ácidos graxos de cadeia curta, alterações na fração entre níveis circulantes de aminoácidos de cadeia ramificada e aromática, além de ácido aminobutírico (GABA), podem se comportar como falsos neurotransmissores (RADINSKY, 2013).

A amônia, produzida no intestino grosso pela degradação bacteriana de ureia, aminoácidos, aminas e purinas é transportada pelo sistema porta para o fígado, onde é convertida em ureia e glutamina. Já o GABA, neurotransmissor inibitório, produzido por bactérias entéricas e habitualmente metabolizado no fígado também apresenta receptores com locais de ligação para barbitúricos, benzodiazepínicos e substâncias similares aos benzodiazepínicos, os ligantes. Quando os receptores benzodiazepínicos endógenos se ligam ao complexo de receptores de GABA, pode ocorrer uma depressão do sistema nervoso central. Estes ligantes estão circulantes em níveis elevados em cães com DPSC (TOBIAS, 2007). 
Outras substâncias encontradas em níveis excessivos circulantes incluem benzodiazepínicos endógenos, triptofano, ácidos biliares, falsos neurotransmissores e outros. Estas substâncias podem impedir a função dos neurónios e dos astrócitos, provocando edema celular, inibição dos canais de íon, elevação intracelular da concentração de cálcio, depressão de atividade elétrica, e interferência com metabolismo oxidativo. Estes efeitos, adicionados a alteração de permeabilidade da barreira hematoencefálica observados na encefalopatia hepática, prejudicam a função cerebral (BERENT; TOBIAS, 2012).

A ocorrência da encefalopatia hepática tem sido correlacionada com constipação, azotemia, uso de medicamentos sedativos ou anestésicos e DL-metionina, sangramento gastrointestinal, hipocalemia, alcalose, hipovolemia, hipoxia, hipoglicemia, refeições ricas em proteína, infecção e transfusão com hemácias armazenadas (TOBIAS, 2007).

Os distúrbios de coagulação que determinam a falência hepática são multifatoriais e dependem da interação entre coagulação, anticoagulação e sistemas fibrinolíticos. As hemorragias espontâneas são incomuns, mas as complicações podem ser induzidas quando associadas com úlceras gastrointestinais, drogas anticoagulantes concomitantes, procedimentos invasivos - cirurgia, aspiração ou biópsia. As causas de coagulopatia com falência hepática incluem diminuição de síntese de fatores (II, V, XII, IX), aumento do uso de fatores, demonstrado pelo aumento de atividade do fator VIII, aumento de fibrinólise e liberação de tromboplastina, síntese anormal de coagulantes (disfibrinogenemia), diminuição da contagem e função plaquetária, deficiência de vitamina K1, e aumento da produção de anticoagulantes (BERENT; TOBIAS, 2012).

\section{Sinais clínicos DPSC}

Cães de raças puras tem risco maior de apresentar DPSCs e gatos domésticos de pelo curto são os mais afetados, embora os da raça himalaio também possam ser portadores. Os DPSCs únicos geralmente congênitos são diagnosticados em cães jovens com dois a três anos, mas há relatos em canídeos com 10 anos de idade. (RADINSKY, 2013).

Os animais com DPSCs podem apresentar sinais relacionados a disfunção hepática que incluem sinais do trato gastrointestinal como anorexia, depressão, perda de peso, vômitos, diarréia, melena, sinais neurológicos compatíveis com encefalopatia hepática como depressão, letargia, ataxia, comportamento anormal, estupor, salivação excessiva (principalmente em gatos) e cegueira, além de retardo no crescimento, poliúria, polidipsia e urolitíase, hematúria e estrangúria associados a litíase urinária. Alguns são assintomáticos e tem o diagnóstico de DPC quando apresentam microhepatia ou hipoalbuminemia (WOLSCHRIJN et al., 2000; MEHL et al., 2005; RADINSKY, 2013). Qualquer paciente jovem com recuperação prolongada após anestesia ou tranquilização pode ser suspeito de DPC, sendo um dos primeiros sinais apresentados em alguns animais (RADINSKY 2013). Outros defeitos congênitos descritos incluem criptorquidismo, em $30 \%$ dos gatos e $50 \%$ dos cães e murmúrios cardíacos, a presença de coloração cobre na íris foi documentada particularmente em gatos (BERENT; TOBIAS, 2009).

Os sinais podem variar, de moderado a grave, como letargia, aparente cansaço e lentidão a mudanças como estupor, andar compulsivo, andar em círculos, amaurose, convulsão e coma (RADINSKY, 2013). A ascite é rara exceto quando há hipoalbuminemia severa, hipertensão portal ou sangramento gastrointestinal (BERENT; TOBIAS, 2009). Mehl et al. (2005) constataram que $82 \%$ dos cães com DPS intrahepático apresentavam sinais neurológicos centrais, $76 \%$ sinais gastrointestinais e $39 \%$ sinais urológicos. 


\section{Diagnóstico DPSC}

Alguns cães com DPSC podem ter exames de rotina normais. Entretanto é comum o encontro de eritrócitos microcíticos e normocrômicos, anemia não regenerativa moderada, células alvo ou poiquilocitose. Pode ocorrer hipoalbuminemia, redução colesterol sérico e/ou ureia sanguínea (pela redução da conversão da amônia em ureia no ciclo da ureia hepática). Aumento moderado ou marcante nas enzimas hepáticas alanina aminotransferase sérica, aspartato aminotransferase, e fosfatase alcalina. A concentração de manganês pode estar elevada em cães com DPSC pois esse elemento é normalmente excretado via conjugação hepática e pode ser implicado na encefalopatia hepática. A bilirrubina sérica geralmente é normal. A hipoglicemia é rara e os tempos de coagulação são geralmente normais. A densidade urinária pode estar baixa ou ocorre a cristalúria com cristais de biurato. Se houver litíase por urato pode haver hematúria, piúria e proteinúria. Os ácidos biliares podem ter valor quando é realizada a mensuração pré e pós-prandial, pois cerca de $20 \%$ dos cães acometidos pela DPSC apresentam uma elevação dos ácidos biliares no período pós-prandial, porém nem sempre estarão manifestando sinais clínicos importantes. Podem ter baixo nível de proteína C, o que ocorre também em doenças hepatobiliares. A hiperamonemia é muito específica para insuficiência hepática, mas sua mensuração simples não é sensível mesmo em encefalopatia hepática. Por isso deve ser medida depois de seis a oito horas pós prandial, e processada logo após a colheita (RADINSKY, 2013; MANKIN, 2015).

A maioria dos DPSC extra-hepáticos termina na veia cava caudal, cranial à veia renal junto ao forame epiploico. Porém, podem acompanhar a curvatura menor do estômago e terminar na veia frênica ou veia hepática esquerda cranial ao fígado. Os desvios porto-ázigos geralmente são localizados na altura do diafragma ao nível da crura ou hiato aórtico. É raro um segundo vaso anômalo além do DPSC nas regiões citadas num mesmo animal. Ocasionalmente o diagnóstico de DPSC extra-hepático pode ser feito durante a laparotomia exploratória se o veterinário for familiarizado com a anatomia abdominal. Já os DPSCs intra-hepáticos são mais difíceis de serem detectados durante uma cirurgia exploratória (BERENT; TOBIAS, 2009).

O diagnóstico por imagem do DPC em cães e gatos pode ser efetuado com o emprego de diferentes técnicas, contudo as mais utilizadas são; portografia mesentérica intraoperatória, portografia retrógrada, portografia esplênica transabdominal, ultrassonografia abdominal com doppler pulsado e colorido cintilografia portal via retal, cintilografia portal transabdominal por injeção de 99m Tc macroagregados na veia lienal guiada por ultrassom, angiografia por ressonância magnética (SANTILLI; GERBONI, 2003) e por tomografia computadorizada (ZWINGENBERGER et al., 2005).

Os exames por imagem permitem a identificação do animal portador de DPSC, com a visualização da morfologia do vaso anômalo que permite a orientação da intervenção cirúrgica que será realizado, reduzindo assim a morbidade e a mortalidade do procedimento. Em cães suspeitos com DPSC, as radiografias geralmente são irrelevantes com exceção da redução de volume hepático e alargamento renal e se for utilizada a angiografia pela tributária da veia porta, com a introdução de agulha intravenosa na veia jejunal. Outras técnicas angiográficas podem ser realizadas como a portografia mesentérica intraoperatória ou retrógrada via cateter na veia hepática direita. A ultrassonografia é considerada o método de escolha para confirmar DPSC em cães e gatos e permite de forma não invasiva, a avaliação vascular do fígado e anormalidades do trato urinário associados a esta afecção. Possui sensibilidade de $80,5 \%$ em casos DPSC extra-hepático em cães e especificidade de $66.7 \%$ e sensibilidade de $100 \%$ para DPSC intra-hepático. 0 uso da técnica do doppler colorido e espectral aumenta o valor diagnóstico da ultrassonografia, sendo detectado um fluxo turbulento na veia cava caudal e vaso anômalo. Podese avaliar também a hemodinâmica portal. Em casos de DPSC, o diâmetro da veia porta é reduzido enquanto que o da veia cava caudal ultrapassa o da artéria aorta em $100 \%$ em casos de localização intra-hepática e $88.9 \%$ nos extra-hepáticos em cães. Por outro lado, nos casos de desvios porto-ázigos o diâmetro da veia cava é normal e a veia ázigos apresenta-se alargada e visível na posição crâniodorsal em relação a aorta abdominal. Desta forma a sensibilidade do ultrassom com doppler aumenta 
para $95 \%$, especificidade para 98\%. Entretanto a acurácia do diagnóstico depende de um operador experiente (SANTILLI; GERBONI, 2003).

A tomografia computadorizada propicia uma boa qualidade de imagem do abdômen, sendo utilizada para o estudo do sistema vascular portal e hepático para avaliação da anatomia vascular e dos desvios portossistêmicos nos animais, facilitando, portanto, o trabalho da equipe cirúrgica (BERTOLINI et al., 2006). A angiografia por tomografia computadorizada é um procedimento minimamente invasivo e rápido. Permite a obtenção de imagens de todos os tributários portais e ramos ao serem preenchidos com meio de contraste durante uma injeção venosa periférica única (Figura 1). Este método permite diagnosticar desvios portossistêmicos extra-hepáticos múltiplos e simples, além de desvios portossistêmicos intra-hepáticos (ZWINGENBERGER et al., 2005).

Figura 1. Angiotomografia do abdome de um cão, (A) corte transversal e (B) reformatação em plano sagital, mostram um desvio portossistêmico portocava. AO (aorta), VCC (veia cava caudal), VP (Veia porta)

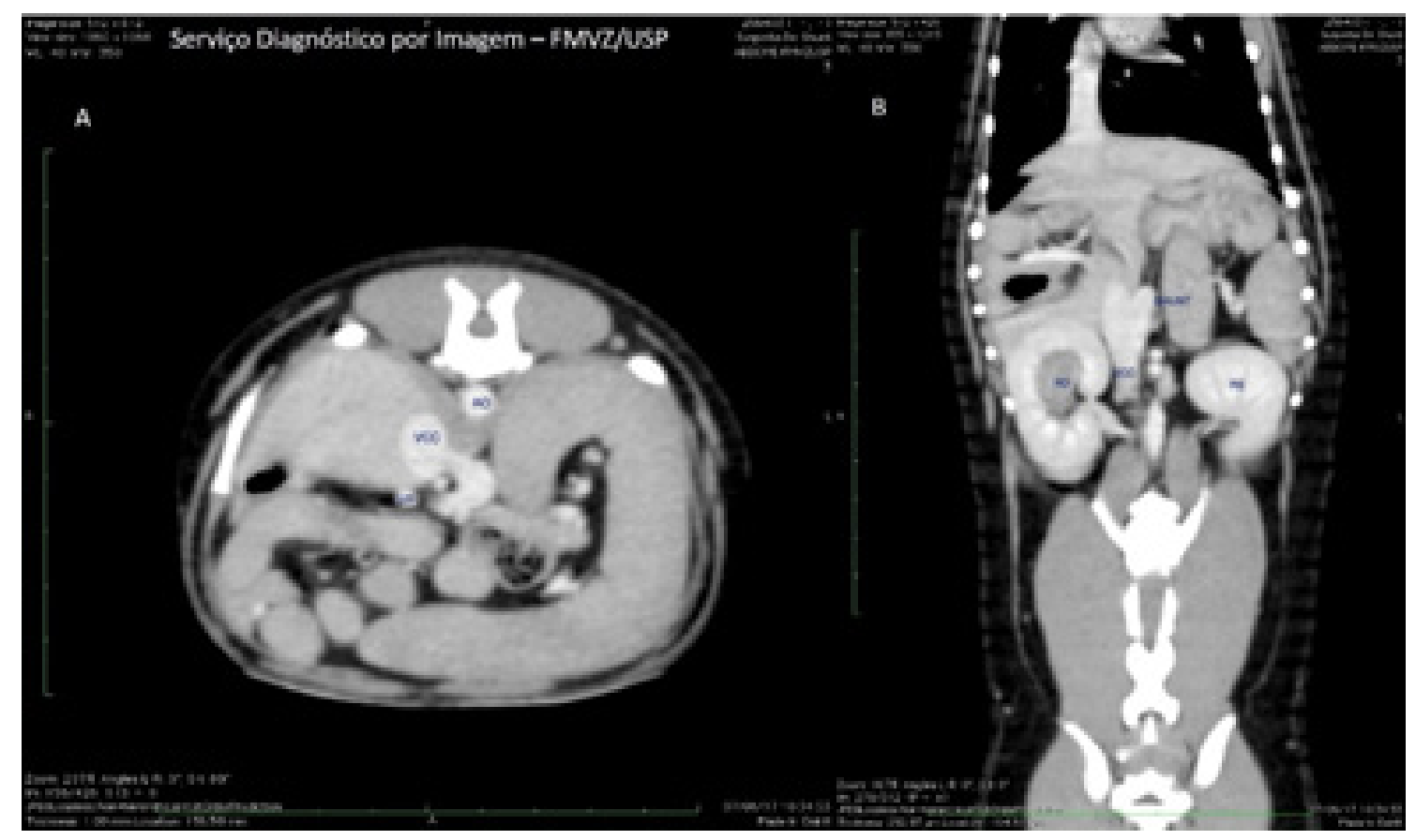

T Fonte: Serviço de Diagnóstico por imagem do Depto de Cirurgia da FMVZ-USP (2019).

A angiografia com tomografia computadorizada permite o detalhamento da anatomia do desvio porto-ázigos em cães. Existem poucas variações anatômicas de tipos de desvios. A maioria dos cães com desvio portoázigos possui um desvio gastroázigo esquerdo, enquanto que outros envolvem a veia gástrica direita. Estes dados são importantes, pois a identificação de desvio portoázigo é um desafio cirúrgico e pode ser difícil a colocação de dispositivo oclusor cranial a todos os ramos gástricos (OR et al., 2016). Os tipos mais comuns de DPSCs extra-hepáticos e as suas conexões foram descritos por Bertolini (2019) (Figura 2). 
Figura 2: Esquema proposto dos principais vasos envolvidos no desvio portossistêmico congênito (DPSC) extra-hepático mais comum direito (D) e esquerdo (E), sendo que as linhas vermelhas pontilhadas seriam as conexões normais entre veia porta e suas tributárias

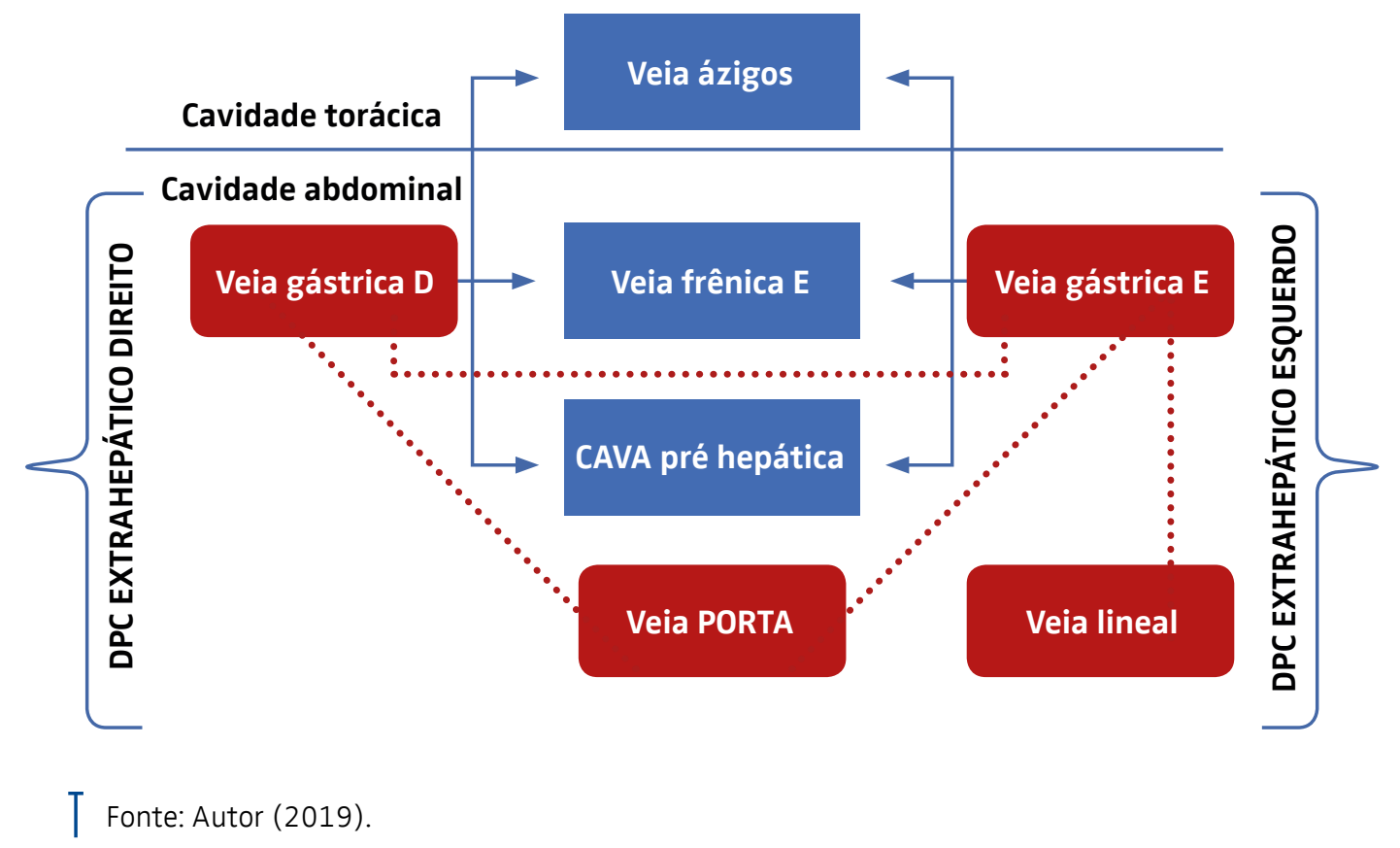

\section{Diagnóstico diferencial}

É importante realizar o diagnostico diferencial do DPSC com outras afecções que determinam insuficiência hepática, como a cirrose, e anormalidade neurológicas comuns em raças miniaturas como a hidrocefalia, meningoencefalite infecciosas ou inflamatórias, epilepsia. As radiografias abdominais na procura de micro-hepatia e os testes de função hepática como ácidos biliares pré e pós-prandiais determinam o painel de exames para DPSC. Se os resultados obtidos em tais exames forem normais, o DPSC ainda é possível, mas menos provável e, portanto, outras doenças devem ser consideradas (RADINSKY, 2013). Em cães de porte grande, outro diagnóstico diferencial importante é a malformação arteriovenosa, que pode determinar os mesmos sinais clínicos e consiste em um vaso aberrante que sai de uma artéria hepática e entra na veia porta-hepática devido a um desenvolvimento anormal da vasculatura hepática. As alterações hemodinâmicas levam a uma turbulência na malformação e a um aneurisma. A consequência é uma hipertensão portal e desvios portossistêmicos adquiridos (TAPPIN; RIZZO, 2007). Outra forma de classificação (Tabela 1) descrita recentemente são os colaterais portais categorizados de alto fluxo: malformações hepáticas arterioportal, fístula arteriovenosa e baixo fluxo: DPSC extrahepáticos latero-terminal (ausência congênita da veia porta) e latero-lateral (portocaval e portoázigos, provenientes da veia gástrica esquerda ou direita) e outras anomalias vasculares congênitas e adquiridas descritas são ausência da veia porta e trombose da veia porta (BERTOLINI, 2019). 
Tabela 1: Resumo de classificação proposta por Bertolini (2019) das conexões anômalas envolvendo o sistema venoso porta e anomalias da veia porta

\begin{tabular}{l|l}
\hline Conexões anômalas envolvendo sistema venoso porta & Anomalia veia porta \\
\hline
\end{tabular}

\section{Alto fluxo}

- Malformação arteriovenosa hepática (congênita)

- Fístula arteriovenosa (adquirida)

- Ambos podem ter relação com desvio portossistêmico adquirido e anomalias colaterais

\section{Baixo fluxo}

- Desvio portossistêmico congênito

- Desvio intra-hepático

- Desvio extra-hepático

- Término-lateral (+ausencia congênita veia porta)

- Látero-lateral (origem da veia gástrica esquerda ou direita)

- Portocaval

- Portoázigos

- Desvio portossistêmico adquirido

- Grandes

- Varizes

- Colaterais porto-portal

- Colaterais cavo-portal
A. Ausência congênita da veia porta (aplasia/atresia) que pode ter relação com desvio extra-hepático termino lateral

B. Hipoplasia da veia porta

- Hipoplasia veia porta primária que pode ter relação com grandes desvios portossistêmico adquiridos

- Hipoplasia veia porta secundária que pode ter relação com desvio portossistêmico congênito laterolateral

C. Aneurisma veia porta (congênita/ adquirida) que pode originar a trombose

D. Trombose veia porta (parcial/ completa) que pode ter relação com grandes desvios portossistêmico adquiridos e colaterais cavo-portais

Bertolini (2019).

\section{Tratamento DPC}

Há duas sugestões possíveis para o tratamento do DPSC - redirecionar cirurgicamente o sangue através do parênquima do fígado geralmente pela atenuação do vaso anômalo ou minimizar a carga de fatores originados pelo trato gastrointestinal entrando na circulação sistêmica com o emprego de manejo clínico. Tais procedimentos também podem ser associados. 0 tratamento cirúrgico seria indicado para animais portadores de DPSC com sinais clínicos mais estáveis. 0 tratamento médico para os sintomáticos, visa a identificação e correção dos fatores predisponentes para encefalopatia hepática, reduzindo a absorção de toxinas produzidas pelas bactérias intestinais e possivelmente diminuindo a lesão oxidativa dos hepatócitos. Evitar dieta com alto teor de proteína, infecção bacteriana, principalmente trato urinário, sangramento intestinal, transfusão sanguínea, fármacos impróprios (RADINSKY, 2013; GREENHALGH et al., 2010).

Podem-se instituir dietas comerciais para hepatopatas ou doenças intestinais ou dieta caseira com proteína de alta qualidade e digestão como frango e queijo cottage associado a arroz ou massa (GREENHALGH et al., 2010). Antibioticoterapia oral ou parenteral para reduzir a flora entérica para 
evitar toxinas como a amônia é indicada como por exemplo o metronidazol 10mg/kg BID ou TID ou ampicilina $20 \mathrm{mg} / \mathrm{kg}$ BID. A lactulose utilizada para acidificar conteúdo do cólon, diminuir o tempo de trânsito intestinal e reduzir a produção e absorção de amônia, pode ser dada pela via oral $(0,5-1 \mathrm{ml} /$ $\mathrm{kg}$ tid a quid) ou enema de retenção (30\% solução lactulose a 5 a $10 \mathrm{ml} / \mathrm{kg}$ ). Se em coma hepático o tratamento deve ser agressivo, com enemas de água morna para lavagem e em seguida o enema de retenção com lactulose, correção eletrólitos e glicemia. Pode ocorrer lesão oxidativa nos hepatócitos, portanto o uso de antioxidantes pode auxiliar na proteção da membrana como o S-adenosil-Lmetionina (Same) e silimarina. Além disso o ácido ursodeoxicólico também ajuda a proteger a membrana do hepatócito (BERENT; TOBIAS, 2009; RADINSKY, 2013). Em coagulopatias sintomática ou pós-operatória administrar plasma fresco congelado $10-15 \mathrm{ml} / \mathrm{kg}$ por 2 a 3 horas, vitamina $\mathrm{K} 11.5-2 \mathrm{mg} / \mathrm{kg}$ via subcutânea ou intramuscular BID por 3 doses, após SID (BERENT; TOBIAS, 2009).

O tratamento medicamentoso ou conservador é apenas paliativo (BUSSODORI et al., 2008), pode aliviar sinais clínicos de encefalopatia hepática, porém a longo prazo é questionável pois o fluxo sanguíneo portal continua desviado para a circulação sistêmica, comprometendo a distribuição de fatores hepatotróficos ao fígado e ocorrendo uma contínua atrofia do órgão (HUNT; HUGHES, 1999). 0 único tratamento definitivo é o cirúrgico ou a oclusão intervencionista do vaso anômalo (BUSSODORI et al., 2008). A completa eliminação do shunt portossistêmico é indicada para a resolução dos sinais clínicos e aumento da sobrevida do animalo (ADIN et al., 2006). Entretanto, a avaliação pré-cirúrgica deve analisar se os animais estão caquéticos, com encefalopatia ou instáveis e, portanto, necessitando de manejo médico para tolerarem o estresse da anestesia e da cirurgia. Se apresentarem baixa condição corpórea, deverão adquirir peso antes da realização da cirurgia. A anestesia deve ser induzida com opioide, propofol ou máscara de isofluorano ou sevofluorano com oxigênio, mantida como os mesmos anestésicos e com controle da glicemia (BERENT; TOBIAS, 2013).

Estudo recente avaliou 78 prontuários de cães portadores de um único desvio porto-sistêmico congênito que receberam tratamento não cirúrgico, com ou sem lactulose e ambos com dieta de restrição proteica. Acesso telefônico foi positivo com 37 tutores destes cães que responderam ao questionário de qualidade de vida. A média de vida estimada foi de 3,2 anos, sem diferenças entre desvios extra $(n=48)$ ou intra-hepático $(n=29)$ e tampouco com ou sem adição de lactulose. A qualidade de vida durante o período de tratamento conservador apresentou melhora significativa $(p<0.01)$. Conclui-se que quando a opção cirúrgica não é viável, o tratamento clínico pode ser uma boa opção de melhora de qualidade de vida. No entanto, há necessidade de realização de mais estudos prospectivos com tratamento clínico relacionados aos diferentes tipos de localização dos vasos anômalos (FAVIER et al., 2020). GREENHALGH et al. (2014) avaliaram a longo prazo a qualidade de vida em cães operados $(n=97)$ ou não $(n=27)$ portadores de DPSCs. Dentre estes 45 cães morreram ou foram eutanasiados, dos quais 24 após tratamento clínico e 21 após cirurgia durante o período avaliado. A taxa de sobrevivência foi significativamente maior em cães submetidos a cirurgia do que nos que receberam somente a tratamento clínico. A frequência de sinais clínicos foi menor em cães operados do que não operados. De um modo geral conclui-se que o tratamento cirúrgico resultou em aumento da taxa de sobrevivência e menor taxa de sinais clínicos posteriores e que a idade no diagnóstico não afetou a taxa de sobrevivência e não deve influenciar o tratamento de escolha.

O tratamento cirúrgico dos DPSCs extra-hepáticos congênitos consiste na sua atenuação para normalizar a circulação sanguínea no sistema porta. As técnicas descritas incluem atenuação com fio de seda (HUNT; HUGUES, 1999), banda de celofane (CASE et al., 2016; HUNT; HUGUES, 1999; HUNT et al., 2004), ameroide constrictor (MEHL et al., 2006; SEREDA; ADIN, 2005), embolização transvenosa com coil (CASE et al., 2016; BUSSADORI et al., 2008; LÉVEILLÉ; JOHNSON; BICHARD, 2003), oclusor hidráulico percutâneo (ADIN et al., 2006).

O ameroide constrictor (Figura 3) atualmente usado para o tratamento cirúrgico de DPC contém no seu interior um anel dentro de uma cobertura externa de aço inoxidável. Um pequeno fragmento cilíndrico funciona como uma chave que fecha o anel após a sua colocação ao redor do vaso anômalo. 
À medida que o ameroide absorve fluídos, ocorre uma expansão centrípeta dentro do anel de aço inoxidável, resultando em um decréscimo da área luminal interna. Na dependência do tamanho, formato, dureza do material revestindo o ameroide, assim como tipo e temperatura do fluido subjacente altera a taxa de fechamento, tem sido demonstrado um padrão de expansão consistente, ocorrendo mais rapidamente nos primeiros três a 14 dias após a colocação, e depois de forma mais vagarosa (SEREDA; ADIN, 2005).

Figura 3: A - Imagem de um dispositivo de ameroide constrictor, com sua camada externa de aço inoxidável e interna de material expansivo (verde) após contato com líquidos corporais e trava/chave. B - Imagem transoperatória após ser inserido o dispositivo ao redor do vaso anômalo

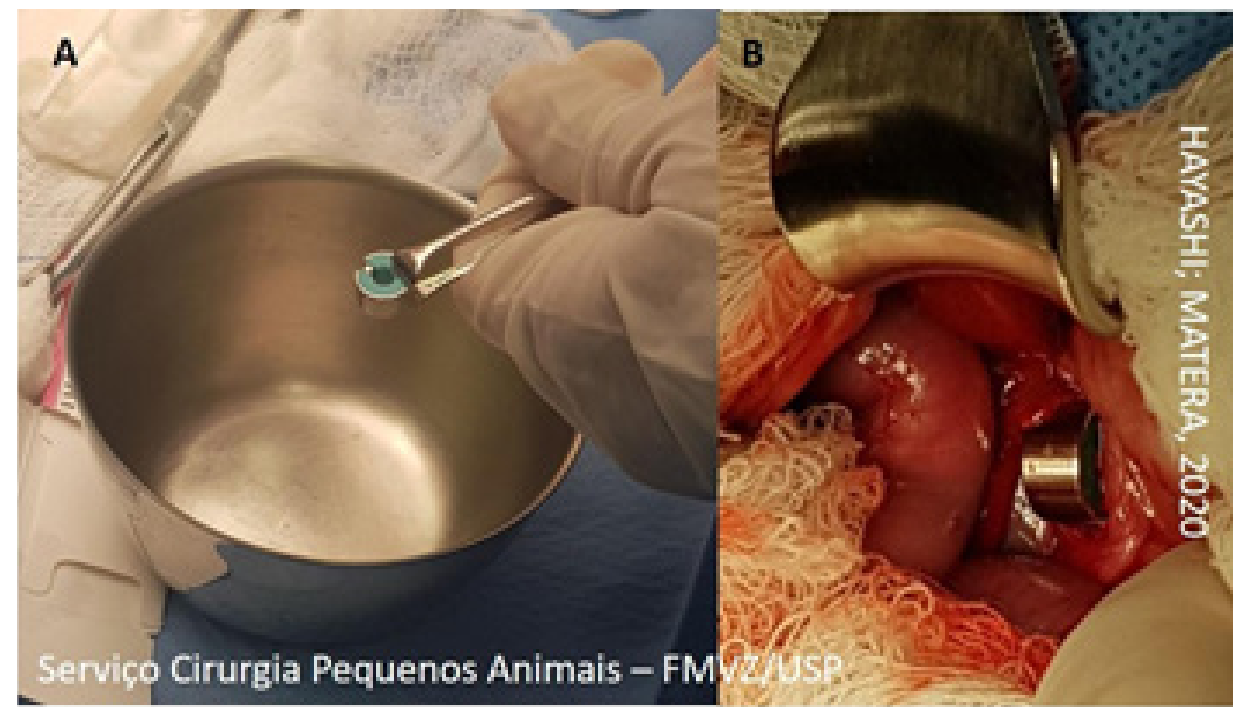

T Fonte: Serviço de Cirurgia de Pequenos Animais- FMVZ/USP(2020).

Já a banda de celofane colocada em posição extravascular produz uma reação a corpo estranho crônica similar ao ameroide, ocorrendo oclusão vascular progressiva. Sereda e Adin (2005) sugerem que a atenuação inicial do vaso anômalo de $3 \mathrm{~mm}$ seria o diâmetro interno máximo que progrediria para a completa oclusão. Hunt et al. (2004) analisaram retrospectivamente os casos de 95 cães e cinco gatos com DPSC extra-hepáticos e de 11 cães com DPSC intra-hepáticos em que a atenuação foi realizada com o emprego da banda de celofane e concluíram que o procedimento foi seguro e efetivo podendo ser uma alternativa para outros métodos de atenuação. Da mesma forma para DPSC extra-hepáticos, a atenuação pela colocação de banda de celofane (tira de três camadas e largura de $4 \mathrm{~mm}$ ) foi usada e não necessitou de diâmetros menores do que $3 \mathrm{~mm}$ para a obtenção de resultados favoráveis (FRANKEL et al., 2006).

A embolização transvascular é realizada pela colocação de um material trombogênico no lúmen do vaso via cateter e fio guia. A oclusão do lúmen do vaso ocorre assim que se forma um trombo ao redor e no material embolizante. Coils são manufaturados de uma tira metálica flexível, de aço inoxidável ou platinum, e fibras de poliéster múltiplas que podem ser depositadas com o emprego de um cateter e que estimulam a trombose. Podem ocorrer complicações como migração do material ou necessidade de procedimentos múltiplos (SEREDA; ADIN, 2005). Weisse et al. (2014) avaliaram a colocação de stent na veia cava - na região do orifício do vaso anômalo, associado ao coil trombogênico para evitar as complicações como migração e com boa taxa de sucesso. Culp et al. (2018) obtiveram resultados positivos em 22 de 24 cães submetidos a embolização percutânea transvenosa em cães com DPCs intra-hepáticos. Foram acompanhados ao redor de três meses de pós-operatório, com melhora de até $50 \%$ dos exames laboratoriais e resolução dos sinais clínicos. Em 15 cães que puderam ser avaliados 
por mensuração do fluxo por doppler, foi constatado aumento de fluxo sanguíneo portal para o fígado com diminuição da fração arterial hepática.

O oclusor hidráulico de silicone via percutânea usado em dez cães com DPC intra-hepático foi efetivo para o tratamento cirúrgico. A revisão do implante foi necessária em três cães devido a ruptura $(n=2)$ ou deslocamento do tubo $(n=1)$. Todos os cães tiveram melhora dos sinais clínicos. Não foi observada recorrência de sinais em oito cães após uma média de 22 meses após a cirurgia. Contudo, alguns problemas constatados com a confiabilidade do implante indicaram a necessidade de modificações na sua forma de fabricação (ADIN et al., 2006). No entanto, Wallace et al. (2018) descreveram $100 \%$ de sucesso $(n=6)$ no uso de dispositivo radioluscente de silicone, que promove oclusão gradual e autoestático, porém apenas a diâmetros comparáveis de 3,5mm de um ameroide constrictor. A completa atenuação foi observada em quatro pacientes e parcial em dois, com fluxo residual médio em oito semanas de pós-operatório.

Apesar de ainda faltarem mais estudos, os dados atuais indicam que o método ideal de oclusão vascular gradual ainda não foi identificado (SEREDA; ADIN, 2005). Uma revisão sistemática e metaanálise avaliou 48 trabalhos que tinham critério de inclusão (de um total de 631 encontrados) sobre o tratamento de DPSCs extra-hepáticos em cães. Concluíram que a base de evidência de escolha de tratamento nestes cães ainda é fraca apesar de recentes publicações. 0 dispositivo de ameroide constrictor é superior ao filme de celofane, no entanto, faltam estudos randomizados e cegos para as diferentes modalidades e com a rotina de incluir imagens de pós-operatório para acessar o fechamento do vaso anômalo e desenvolvimento de desvios porto-sistêmicos adquiridos (SERRANO et al., 2019).

A ligadura completa do vaso anômalo acarreta a alteração dos parâmetros hemodinâmicos e determina risco de hipertensão portal aguda e morte (WOLSCHRIJN et al., 2000). Além disso, a ligadura parcial deixa um fluxo residual relativamente alto, portanto, não sendo muito utilizada quando se compara aos outros métodos de atenuação gradativa como os ameroides constrictores (WOLSCHRIJN et al., 2000; BUSSADORI et al., 2008). A avaliação transoperatória de um possível aumento de pressão portal, é baseada na alteração da frequência cardíaca, pressão arterial sistêmica, motilidade e cor do intestino (TISDALL et al., 2000).

As técnicas de cirurgias abertas para atenuação dos vasos anômalos são realizadas por uma celiotomia de rotina na linha média ventral abdominal. Aconselha-se cuidado, pois pode ser seccionado um vaso anômalo que porventura esteja dentro do tecido gorduroso do ligamento falciforme. Além disso, a cuidadosa análise dos resultados do diagnóstico por imagem permitirá a correta localização do vaso anômalo no transoperatório, e facilitará a atuação do cirurgião (MANKIN, 2015).

A escolha do local de colocação para fechamento ou atenuação do vaso anômalo está implicada com a morfologia do desvio porto-sistêmico extra-hepático. White, Parry e Shales (2018) avaliaram 54 cães e 10 gatos que apresentaram cinco tipos distintos de desvios: gastrofrênico esquerdo, gastrocaval direito, esplenocaval, colocaval e gastro-ázigos esquerdo. A tomografia computadorizada com angiografia e as observações macroscópicas intraoperatórias confirmaram quatro locais consistentes de comunicação entre o vaso anômalo e o sistema venoso sistêmico: veia cava caudal na altura do forame epiplóico, veia frênica esquerda na altura do hiato esofágico, veia ázigos na altura do hiato aórtico e veia cava caudal ou veia ilíaca na altura da sexta ou sétima vértebra lombar. 0 uso da portografia intraoperatória foi efetiva para confirmar que durante a cirurgia todos os vasos portais tributários eram proximais ao ponto de atenuação do vaso anômalo.

A morfologia do DPSC intra-hepático determina em grande parte a viabilidade e a técnica que será usada para o tratamento cirúrgico. 0 duto venoso patente (DPSC intra-hepático esquerdo) pode ser tratado pela atenuação da veia hepática esquerda ou pela ligadura do vaso anômalo assim que entra na veia hepática esquerda na superfície diafragmática do lobo hepático medial ou lateral esquerdo. Por outro lado, o desvio que passa pelo lobo hepático medial ou lateral direito é de difícil abordagem cirúrgica e uma variedade de técnicas foram descritas: dissecção pré-hepática das tributárias portais, dissecção do parênquima hepático circundante, e abordagem intravascular via veia cava caudal ou veia porta (LAMB; WHITE, 1998). 
Tobias, Byarlay e Henry (2004) descreveram uma passagem indireta para a ligadura com fio de seda 2-0 ou polipropileno 0 da veia porta direita realizada com sucesso em portadores de DPSC, sendo viável a realização de atenuação com dispositivos constrictores ao invés da ligadura. As vantagens são tempo reduzido e risco limitado de hemorragia e perfuração vascular.

Quando o vaso anômalo não oclui completamente, nos casos onde há um grande volume de sangue pela circulação portal hepática hipoplásica pode ocorrer a hipertensão portal. A completa atenuação foi relatada em somente 11 a 55\% de desvios extra-hepáticos em cães. As taxas de mortalidade associadas a correção cirúrgica de desvios porto-sistêmicos de todos os tipos variaram de 14 a 50\%, sendo a causa da morte por hipertensão portal, hemorragia, convulsões intratáveis após recuperação anestésica, o que denominam de síndrome da convulsão pós ligadura (HUNT; HUGHES, 1999). No entanto, de uma forma geral, o uso de ameroides constrictores em 206 cães com DPSCs extra-hepáticos revelou que $7 \%$ dos pacientes morreram em 30 dias, $24 \%$ tem o desvio persistente e 92\% não manifestam sinais clínicos (MANKIN, 2015).

Caporali (2015) avaliou 95 cães portadores de DPSC extra-hepáticos e encontrou urólitos em $35,8 \%$ dos animais $(n=34)$, sendo que dos 17 urólitos analisados, 16 eram de urato de amônio. Cães machos, cães idosos e cães recebendo tratamento médico prévio são considerados de risco para o desenvolvimento de urólitos.

Após atenuação cirúrgica do DPSC pode ocorrer recorrência de sinais de encefalopatia hepática. Estes sinais neurológicos decorrem de desvios múltiplos adquiridos após ligação completa do vaso anômalo. (HUNT: HUGHES, 1999; TISDALL et al., 2000). Além disso, sinais clínicos de hipertensão portal podem ocorrer após a oclusão parcial ou total do desvio ou formação de trombo (BESACON et al., 2004), e incluem a presença de ascite e melena (TOBIAS; BYARLAY; HENRY, 2004).

Os portadores de DPSCs podem apresentar baixa atividade dos fatores de coagulação, como o prolongamento do tempo da tromboplastina ativada. Este fato pode provocar complicações pósoperatórias como hemorragias graves pós-operatórias após a atenuação do vaso anômalo. Kummeling et al. (2006), analisando o comportamento 30 cães portadores de DPSC, constataram que as alterações que ocorreram imediatamente após a atenuação deste vaso foram: diminuição da contagem plaquetária e da atividade dos fatores I, II, V, VII, IX, X e XI, bem como, prolongamento do tempo da protrombina, alterações que não se normalizaram mesmo após seis semanas do ato cirúrgico. A hemostasia só se normalizou após completa recuperação do shunt após atenuação comparado com cães que ainda não haviam se recuperado.

Hayashi et al. (2019) observaram que os níveis de fibrinogênio séricos baixos $(169 \pm 14.7 \mathrm{mg} / \mathrm{dL}$, referência 200-400) em três cães com DPS extra-hepático foram relacionadas a eventos hemorrágicos

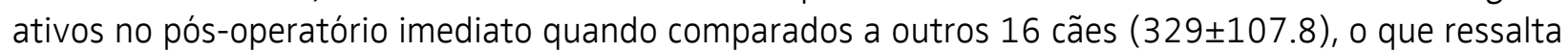
a relevância clínica para a mensuração do nível de fibrinogênio como fator de risco para a ocorrência distúrbios de sangramentos no pós-operatório.

As consequências fisiológicas dos desvios intra ou extra-hepáticas são idênticas, pois o sangue portal faz uma via que evita o fígado, que ocorre por meio de um ducto venoso patente ou de um vaso anômalo extra-hepático. A severidade dos dois tipos de desvios e a sua elevada frequência de ocorrência em várias raças indicam a necessidade de uma urgente avaliação dos aspectos genéticos envolvidos com os desvios portossistêmicos e com a busca de um teste genético confiável para essa anomalia (VAN DEN BOSSCHE; STEENBEEK, 2016).

\section{Considerações finais}

Devido às manifestações neurológicas graves e à necessidade do correto diagnóstico de DPSCs em pequenos animais, determinando o tratamento adequado, é de relevância clínica a correta abordagem para que a intervenção cirúrgica, quando possível, decorra sem maiores complicações, tanto 
na localização do vaso anômalo, bem como minimizando a mortalidade perioperatória. Ressalta-se a importância da equipe multidisciplinar de trabalho - radiologista, clínico e cirurgião no diagnóstico e planejamento do melhor tratamento para esta afecção vascular que determina tantas repercussões sistêmicas.8

\section{Referências}

ADIN, C. A.; SEREDA, C. W.; THOMPSON, M. S. et al. Outcome associated with use of a percutaneous controlled hydraulic occluder for treatment of dogs with intrahepatic portosystemic shunts. Journal of American Veterinary Medical Association, v. 229, p. 1749-1755, 2006. Disponível em: https://doi. org/10.2460/javma.229.11.1749. Acesso em: 29 ago. 2020.

BERENT, A. C.; TOBIAS, K. M. Portosystemic vascular anomalies. Veterinary Clinics Small Animal, v.39, p. 513-541, 2009.

BERENT, A. C.; TOBIAS, K. M. Hepatic vascular anomalies. In: TOBIAS, K. M.; JOHNSTON, S. A. Veterinary surgery: small animal. Canada: Elsevier, v. 2, p. 1624-1658, 2012. Disponível em: https://doi. org/10.1016/j.cvsm.2009.02.004. Acesso em 29 ago. 2020.

BERTOLINI, G. Anomalies of the portal venous system in dogs and cats as seen on multidetector-row computed tomography: an overview and systematization proposal. Veterinary Sciences, v. 6, n. 1, p. 1-17, 2019. Disponível em: https://doi.org/ 10.3390/vetsci6010010. Acesso em: 29 ago. 2020.

BERTOLINI, G. et al. Three-dimensional multislice helical computed tomography techniques for canine extra-hepatic protosystemic shunt assessment. Veterinary Radiology \& Ultrasound, v. 47, n. 5, p. 439-443, 2006. Disponível em: https://doi.org/10.1111/j.1740-8261.2006.00173.x. Acesso em: 29 ago. 2020.

BESANCON, M. F. et al. Evaluation of the characteristics of venous occlusion after placement of an ameroid constrictor in dogs. Veterinary Surgery, v. 33, p. 597-605, 2004. Disponível em: https://doi. org/10.1111/j.1532-950x.2004.04082.x. Acesso em: 29 ago. 2020.

BUSSADORI, R. et al. Transvenous coil embolization for the treatment of single congenital portosystemic shunts in six dogs. The Veterinary Jounal, v. 176, p. 221-226, 2008. Disponível em: https://doi. org/10.1016/j.tvjl.2007.02.027. Acesso em: 29 ago. 2020.

CAPORALI, E. H. G. et al. Risk factors for urolithiasis in dogs with congenital extrahepatic portosystemic shunts: 95 cases. Journal of American Veterinary Medical Association, v. 246, p. 530-536, 2015. Disponível em: https://doi.org/10.2460/javma.246.5.530. Acesso em: 29 ago. 2020.

CULP, W. T. N. et al. Prospective evaluation of outcome of dogs wiht intrahepatic portosystemic shunts treated via percutaneous transvenous coil embolization. Veterinary Surgery, v. 47, p. 74-85, 2018. Disponível em: https://doi.org/10.1111/vsu.12732. Acesso em: 29 ago. 2020.

FAVIER, R. P. et al. Outcome of non-surgical dietary treatment with or without lactulose in dogs with congenital portosystemic shunts. Veterinary Quarterly, v. 40, n.1, p. 108-114, 2020.

FRANKEL, D et al. Evaluation of celofane banding with and without intraoperative attenuation for treatment of congenital extrahepatic portosystemic shunts in dogs. Journal of American Veterinary Medical Association, v. 228, p. 1355-1360, 2006. Disponivel em: https://doi.org/10.2460/ javma.228.9.1355. Acesso em 20 ago. 2020.

GREENHALGH, S. N. et al. Comparison of survival after surgical or medical treatment in dogs with congenital portosystemic shunt. Journal of American Veterinary Medical Association, v. 236, p.12151220, 2010. 
GREENHALGH, S. N. et al. Long-term survival and quality of life in dogs with clinical signs associated with a congenital portosystemic shunt after surgical or medical treatment. Journal of American Veterinary Medical Association, v. 245, p. 527-533, 2014. Disponível em: https://doi.org/10.2460/ javma.245.5.527. Acesso em: 29 ago. 2020.

HAYASHI, A. M. et al. Fibrinogen levels as a fator for surgical decision-making in dogs with portosystemic shunt. Journal of Veterinary Internal Medicine, Meeting abstracts, v. 33, n. 5, p. 2487, 2019.

HUNT, G. B. et al. Outcomes of celofane banding for congenital portosystemic shunt in 106 dogs and 5 cats. Veterinary Surgery, v. 33, p. 25-31, 2004. Disponível em: https://doi.org/10.1111/j.1532950x.2004.04011.x. Acesso em: 29 ago. 2020.

KUMMELING, A. et al. Coagulation profiles in dogs with congenital portosystemic shunts before and after surgical attenuation before and after surgical attenuation. Journal of Veterinary Internal Medicine, $\mathrm{v}$. 20, p. 1319-1326, 2006. Disponivel em: https://doi.org/10.1892/0891-6640(2006)20[1319:cpidwc 12.0.co;2. Acesso em: 29 ago. 2020.

LAMB, C. R.; WHITE, R. N. Morphology of congenital intrahepatic portocaval shunts in dogs and cats. Veterinary Record, v. 142, p. 55-60, 1998.

MANKIN, K. M. Current concepts in congenital portosystemic shunts. Veterinary Clinics of North America: Small Animal Practice, v.45, p. 477-487, 2015. Disponivel em: https://doi.org/10.1016/j. cvsm.2015.01.008. Acesso em: 29 ago. 2020.

MEHL, M.L.etal. Evaluation ofameroid ring constrictors for treatmentfor singleextrahepatic portosystemic shunts in dogs: 168 cases (1995-2001). Journal of American Veterinary Medical Association, v. 226, p. 2020-2030, 2005. Disponível em: https://doi.org/10.2460/javma.2005.226.2020. Acesso em: 20 ago. 2020.

$\mathrm{OR}, \mathrm{M}$. et al. Determination of porto-azigos shunt anatomy in dogs by computed tomography angiography. Veterinary Surgery, v. 46, p. 1005-1012, 2016. Disponível em: https://doi.org/10.1111/ vsu.12553. Acesso em: 29 ago. 2020.

RADINSKY, M. G. Surgery of the liver. In: FOSSUM, T. W. Small animal surgery. 4. ed. St Louis: Elsevier, p. 584-617, 2013.

RICCIARDI, M. Unusual haemodynamics in two dogs and two cats with portosystemic shunt- implications for distinguishing between congenital and acquired conditions. Open Veterinary Jounal, v. 7, n. 2, p. 86-94, 2017. Disponível em: https://doi.org/10.4314/ovj.v7i2.2. Acesso em 20 ago. 2020.

SANTILLI, R. A.; GERBONI, G. Diagnostic imaging of congenital porto-systemic shunt in dogs and cats: a review. The Veterinary Journal, v.166, p. 7-18, 2003. Disponível em: https://doi.org/10.1016/S10900233(02)00304-0. Acesso em: 20 ago. 2020.

SEREDA, C. W.; ADIN, C. A. Methods of gradual vascular occlusion and their applications in treatment of congenital portosystemic shunts in dogs: a review. Veterinary Surgery, v. 34, p. 83-91, 2005. Disponível em: https://doi.org/10.1111/j.1532-950X.2005.00015.x. Acesso em: 20 ago. 2020.

SERRANO, G. et al. Treatment of congenital extrahepatic portosystemic shunts in dogs: a systematic review and meta-analysis. Journal of Veterinary Internal Medicine, v. 33, p. 1865-1879, 2019. Disponível em: https://doi.org/10.1111/jvim.15607. Acesso em: 20 ago. 2020.

SPECHI, S. et al. Canine and feline abdominal arterioportal communications can be classified based on branching patterns in computed tomographic angiography. Veterinary Radiology \& Ultrassound, $\mathrm{v}$. 59, p. 687-696, 2018. Disponível em: https://doi.org/10.1111/vru.12685. Acesso em: 29 ago. 2020.

TAPPIN, S.; RIZZO, F. Case report: hepatic arteriovenous fistula in a 3 month old Border Collie. UK-Vet Companion Animal, v. 12, n. 1, p. 31-36, 2000. 
TISDALL, P. L. C. et al. Neurological dysfunction in dogs following attenuation of congenital extrahepatic portosystemic shunts. Journal of Small Animal Practice, v. 41, p. 539-546, 2000. Disponível em: https://doi.org/10.1111/j.1748-5827.2000.tb03150.x. Acesso em: 29 ago. 2020

TOBIAS, K. M. Desvios portossistêmicos e outras anomalias vasculares hepáticas. In: SLATTER, D. Manual de cirurgia de pequenos animais. 3. ed. Barueri, SP: Manole, v. 1, p. 727-752, 2007.

TOBIAS, K. M.; ROHRBACH, B. W. Association of breed with the diagnosis of congenital portosystemic shunts in dogs: 2400 cases (1989-2002). Journal of American Veterinary Medical Association, v. 223, p. 1636-1639, 2003. Disponível em: https://doi.org/10.2460/javma.2003.223.1636. Acesso em: 2 set. 2020.

TOBIAS, K. M.; BYARLAY, J. M.; HENRY, R. W. A new dissection technique for approach to right-sided intrahepatic portosystemic shunts: anatomic study and use in three dogs. Veterinary Surgery, v. 33, p. 32-39, 2004. Disponível em: https://doi.org/10.1111/j.1532-950x.2004.04009.x. Acesso em: 29 ago. 2020.

VAN DEN BOSSCHE, L.; STEENBEEK, F. G. Canine congenital portosystemic shunts: disconnections dissected. The Veterinary Journal, v. 211, p. 14-20, 2016. Disponível em: https://doi.org/10.1016/j. tvjl.2015.09.025. Acesso em: 29 ago. 2020.

VOGT, J. C. et al. Gradual occlusion of extrahepatic portosystemic shunts in dogs and cats using the ameroid constrictor. Veterinary Surgery, v. 25, p. 495-502, 1996. Disponível em: https://doi. org/10.1111/j.1532-950X.1996.tb01449.x. Acesso em: 20 ago. 2020.

WALLACE, M.L. et al. Gradual attenuation of a congenital extrahepatic portosystemic shunt with a self-retaining polyacrylic acid-silicone device in 6 dogs. Veterinary Surgery, v. 47, p. 722-728, 2018. Disponivel em: https://doi.org/10.1111/vsu.12797. Acesso em: 29 ago. 2020.

WEISSE, C. et al. Endovascular evaluation and treatment of intrahepatic portosystemic shunts in dogs: 100 cases (2001-2011). Journal of American Veterinary Medical Association, v. 244, p. 78-94, 2014. Disponível em: https://doi.org/10.2460/javma.244.1.78. Acesso em: 20 ago. 2020.

WHITE, R. N.; PARRY, A. T.; SHALES, C. Implications of shunt morphology for the surgical management of extrahepatic portosystemic shunts. Autralian Veterinary Journal, v. 96, p. 433-441, 2018. Disponível em: https://doi.org/10.1111/avj.12756. Acesso em: 29 ago. 2020.

WOLSCHRIJN, C. F. et al. Gauged attenuation of congenital portosystemic shunts: results in 160 dogs and 15 cats. Veterinary Quaterly, v. 22, n. 2, p. 94-98, 2000. Disponível em: https://doi.org/10.1080 101652176.2000.9695032. Acesso em: 29 ago. 2020.

ZWINGENBERGER, A. L.; SCHWARZ, T.; SAUNDERS, G. M. Helical computed tomographic angiography of canine portosystemic shunts. Veterinary Radiology \& Ultrasound, v. 46, n. 1, p.27-32, 2005. Disponível em: https://doi.org/10.1111/j.1740-8261.2005.00005.x. Acesso em: 29 ago.2020. 